\title{
Epidemiologic survey of Kawasaki disease in Inner Mongolia, China, between 2001 and 2013
}

\author{
XIAOMEI ZHANG, YANYAN LIANG, WANYU FENG, XUEWEN SU and HUA ZHU \\ Department of Pediatrics, Inner Mongolia Autonomous Region People's Hospital, \\ Hohhot, Inner Mongolia 010017, P.R. China
}

Received April 7, 2015; Accepted May 5, 2016

DOI: $10.3892 /$ etm.2016.3393

\begin{abstract}
The epidemiologic features of Kawasaki disease (KD) in the Inner Mongolia Autonomous Region of China has not been previously determined, to the best of our knowledge. Therefore, the aim of the present study was to investigate the epidemiology of KD in Inner Mongolia. Clinical data from 518 patients treated for KD in Inner Mongolia between January 2001 and December 2013 were analyzed. The results indicated that the mean annual incidence rate was 3.55 \pm 2.96 per 100,000 children under the age of 5 years between 2001 and 2013. The age at diagnosis ranged between 49 days and 14 years, while the disease occurred more frequently in the spring and summer. In addition, the incidence of coronary artery lesion (CAL) was reported to be $40.2 \%$ in the present survey. KD patients in the Han Chinese ethnic group were more likely to be complicated by CAL, whereas patients with incidence of KD in July were less likely to be complicated by CAL. In conclusion, the incidence of KD was observed to be increasing in Inner Mongolia, while the ethnic group and month of onset may be associated with the incidence of CAL in KD patients.
\end{abstract}

\section{Introduction}

Kawasaki disease (KD), which was first reported by Dr Tomisaku Kawasaki in 1967 (1), is a syndrome of unknown cause and causes systemic vasculitis, with symptoms that include persistent fever, bilateral conjunctival congestionpolymorphous exanthema and, alterations of the lip, oral cavity and peripheral extremities (2). It typically affects children under the age of 5 years. Coronary artery lesion (CAL) is the most severe complication of KD and it has been regarded

Correspondence to: Dr Hua Zhu, Department of Pediatrics, Inner Mongolia Autonomous Region People's Hospital, 20 Zhaowuda Road, Saihan, Hohhot, Inner Mongolia 010017, P.R. China E-mail: zhuhua_dr@163.com

Abbreviations: KD, Kawasaki disease; CAL, coronary artery lesion Key words: Kawasaki disease, epidemiology, incidence as one of the major acquired heart diseases in children (3). Therefore, KD has been a major focus in the field of pediatric cardiology research. Although the introduction of intravenous gamma globulin (IVIG) has effectively reduced the rate of CALs, further research is required to ensure early diagnosis and effective treatment of KD (4). Several epidemiologic surveys of KD have been conducted worldwide since 1970's, and various features of the disease have been revealed, such as disease incidence, seasonality, and the rate of CAL (3). $\mathrm{KD}$ has been reported in numerous countries with a greatly varying incidence, and the highest incidence of KD has been reported in Japan, followed by other East Asian countries and districts surrounding Japan (3). However, the epidemiology of $\mathrm{KD}$ has only been reported in a limited number of regions in China, and to the best of our knowledge, it has not been previously determined in the Inner Mongolia Autonomous Region of China. Therefore, in the present study, a survey was conduced to examine the epidemiologic features of KD in Inner Mongolia between 2001 and 2013.

\section{Materials and methods}

Population. A retrospective study of patients treated for the KD in Inner Mongolia between January 1st, 2001 and December 31st, 2013 was conducted. Regardless of ethnicity, patients with KD who were aged $<18$ years old were identified by the discharge diagnosis code in the records of 17 hospitals providing pediatric medical services in Inner Mongolia, including Inner Mongolia Autonomous Region People's Hospital; Maternal and Child Health Hospital of Inner Mongolia; Inner Mongolia Medical University Affiliated Hospital; Ordos Center Hospital; Chifeng Center Hospital; Affiliated Hospital of Chifeng College; The Second Hospital of Chifeng; Tongliao City Hospital; Affiliated Hospital of Inner Mongolia National University; Hulunbuir City People's Hospital; The First Hospital of Hohhot; Baotou Center Hospital; The Second Hospital of Baotou; Linhe City Hospital; Baogang Hospital of Inner Mongolia; The Fourth Hospital of Baotou; and The First and Second Affiliated Hospital of Baotou Medical College. The medical records of the patients with KD during the 13-year span of the study were reviewed. The 5 th revised edition of the diagnostic criteria for KD that was issued by the Japan Kawasaki Disease Research Committee at the 7 th International Kawasaki Disease 
Symposium in 2002 was used for the diagnosis of KD in all cases (2). In brief, the patients included in the study presented at least 5 of the following 6 clinical manifestations, or at least 4 symptoms along with coronary abnormalities documented by echocardiography or coronary angiography: i) Fever persisting for 5 days or longer (including cases in which the fever has subsided before day 5 in response to treatment); ii) bilateral conjunctival congestion; iii) alterations in the lip and oral cavity areas, including diffuse congestion of oral and pharyngeal mucosa, strawberry-colored tongue or reddening of the lips; iv) polymorphous exanthema; v) alterations in the peripheral extremities, such as reddening of the soles and palms, indurative edema at the initial stages, or membranous desquamation at the fingertips during the convalescent stage; and vi) acute nonpurulent cervical lymphadenopathy (5). Patients who did not fulfill the aforementioned criteria, were diagnosed beyond the specified time period or were not residents of the Inner Mongolia region were excluded from the present study.

Survey implementation. The clinical data collected from the eligible patients included the following: Age, gender, ethnic background, location of residence, dates of KD onset and diagnosis, days between symptom onset and hospital visit, clinical signs and symptoms, cardiac and extracardiac manifestations, laboratory examination results (routine blood examination, erythrocyte sedimentation rate and blood chemical tests), echocardiogram results, treatment and outcome.

Statistical analysis. Parametric data are expressed as the mean \pm standard deviation. Nonparametric analysis was performed using Pearson's $\chi^{2}$ test or Fisher's exact test. Differences between the mean values were compared by t-test or one-way analysis of variance. Single-factor analysis and nonconditional multivariate logistic regression analysis were performed to analyze the risk factors for the development of CAL. A value of $\mathrm{P}<0.05$ was considered to indicate a statistically significant difference. All analyses were performed using SPSS software version 19.0 (SPSS, Inc., Chicago, IL, USA).

\section{Results}

Incidence of KD. A total of $597 \mathrm{KD}$ cases were identified in the medical records of the included hospitals within the study period. Of these, 79 cases were excluded through strict quality control, due to not meeting the study criteria. The remaining $518 \mathrm{KD}$ cases were enrolled in the present study population. The number of patients with KD per year, as well as the determined incidence rates in the surveys, are shown in Fig. 1. Based on the Statistical Yearbook data for Inner Mongolia (6), the mean annual incidence rate of KD was $3.55 \pm 2.96$ per 100,000 children under the age of 5 years between 2001 and 2013, and the incidence displayed an increasing trend over this time period. There were 322 (62.2\%) males and 196 (37.8\%) females, with a gender ratio of 1.64:1, respectively. The majority of included KD patients were Han Chinese. Three minority groups were represented by 81 cases $(15.8 \%)$, including 70 Mongolian, 6 Hui and 5 Manchu patients, and these groups were dispersed throughout the surveyed population.



Figure 1. Number of patients with Kawasaki disease and incidence rate in Inner Mongolia (China) by calendar year.

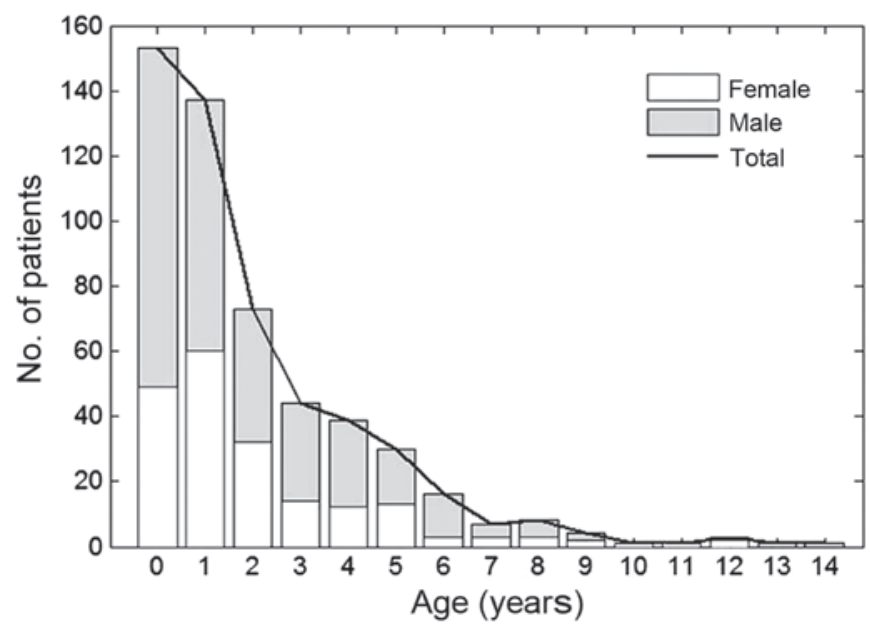

Figure 2. Age distribution of patients with Kawasaki disease in Inner Mongolia (China) between 2001 and 2013.

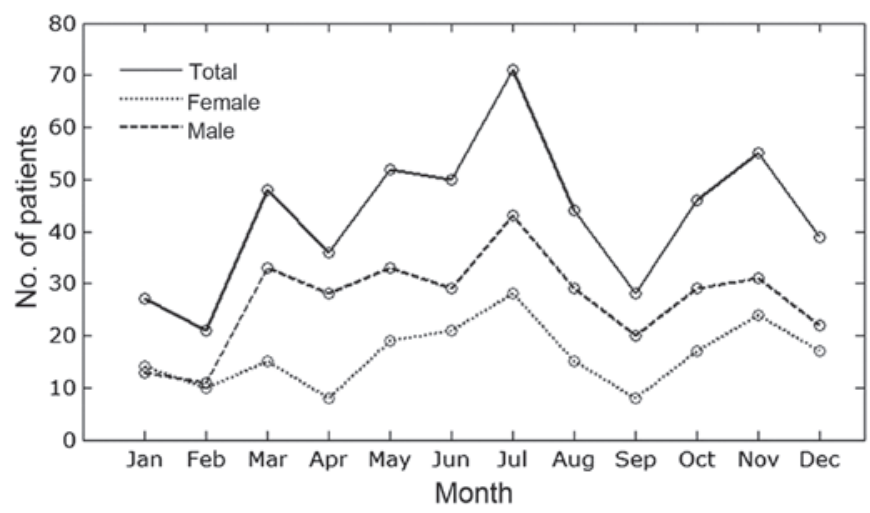

Figure 3. Number of patients with Kawasaki disease in Inner Mongolia (China) according to the month, between the years 2001 and 2013.

Distribution of age at diagnosis of KD. The distribution of age at diagnosis of KD is shown in Fig. 2. The age at diagnosis of KD, which was derived from the date of the first diagnosis (within one month of symptom onset) minus the date of birth, 
Table I. Single-factor analysis of the relevant factors for CAL among the included 518 patients with Kawasaki disease in the Inner Mongolia region between 2001 and 2013.

\begin{tabular}{lcc}
\hline Observed indicator & $\chi^{2}$ test & P-value \\
\hline Age of $\geq 5$ years & 0.245 & 0.620 \\
Gender & 0.099 & 0.782 \\
Ethnic group & 4.657 & $0.031^{\mathrm{a}}$ \\
Month of onset & 21.040 & $0.029^{\mathrm{a}}$ \\
Fever duration of $\geq 5$ days & 1.010 & 0.315 \\
White blood cell count of $\geq 12 \times 10^{9} / 1$ & 0.437 & 0.508 \\
Hemoglobin level of $<100$ g/l & 3.274 & 0.070 \\
Blood platelet count of $\geq 450 \times 10^{9} / 1$ & 1.334 & 0.248 \\
Erythrocyte sedimentation rate of $\geq 40 \mathrm{~mm} / \mathrm{h}$ & 1.170 & 0.279 \\
Creatine kinase-MB level of $\geq 25 \mathrm{U} / 1$ & 0.528 \\
\hline
\end{tabular}

${ }^{\mathrm{a}} \mathrm{P}<0.05$ indicates the observed indicators that were associated with CAL. CAL, coronary artery lesion.

Table II. Nonconditional multivariate logistic regression analysis of Kawasaki disease complicated by coronary artery lesion among the included 518 patients with Kawasaki disease in the Inner Mongolia region between 2001 and 2013.

\begin{tabular}{lccc}
\hline Factor & Partial regression coefficient & OR & 95\% CI \\
\hline Ethnic group (Han Chinese) & 0.736 & 2.087 & $1.083-4.02$ \\
Month of onset (July) & -0.881 & 0.414 & $0.199-0.864$
\end{tabular}

${ }^{\mathrm{a}} \mathrm{P}<0.05$. OR, odds ratio; $\mathrm{CI}$, confidence interval.

ranged between 49 days and 14 years (median, 1.42 years). Cases under the age of 1 year and under the age of 5 years accounted for $56.0 \%$ (290 cases) and $86.1 \%$ (446 cases), respectively, of the patient cohort. The incidence rate was highest among children aged 0-1 years, after which the incidence decreased sharply with increasing age.

Monthly distribution of KD. Trends in the monthly number of patients are shown in Fig. 3. During the survey period, the onset of KD in all patients occurred more frequently in March (48 cases;9.2\%), May (52 cases; 10.0\%), July (71 cases; $13.7 \%$ ) and November (55 cases; $10.6 \%$ ), whereas it occurred relatively less frequently in February (21 cases; 4.1\%). Furthermore, the incidence peak for boys and girls both occurred in July (43 cases, $13.4 \%$ and 28 cases, $14.3 \%$, respectively).

Clinical manifestations of KD. Among the main clinical manifestations of patients with KD in the present study, fever persisting for 5 days or longer was the most frequent manifestation (516 cases; $99.6 \%$ ), followed by changes in the lips and oral cavity (383 cases; $73.9 \%$ ), conjunctival congestion (355 cases; $68.6 \%$ ), polymorphous exanthema (334 cases; 64.5\%), changes in the peripheral extremities at the initial or convalescent stages (322 cases; 62.1\%), and acute nonpurulent cervical lymphadenopathy (321 cases; 61.9\%). Other manifestations included perianal desquamation (118 cases; $22.8 \%$ ), coughing (63 cases; $12.2 \%$ ), diarrhea (17 cases; $3.3 \%$ ), and emesis (12 cases; $2.3 \%$ ). Respiratory system disorders were identified in 186 cases
(35.9\%) and liver impairments were observed in 20 cases (3.9\%). Mortality did not occur in any of the cases during the acute and subacute stages of the disease.

Echocardiographic findings of KD. Echocardiographic examinations were performed in 501 cases (96.7\%) within one month after the onset of KD. Among these patients, abnormal findings were observed in 230 cases $(45.9 \%)$, including coronary artery ectasia (203 cases; 40.5\%), coronary artery aneurysm (2 cases; $0.87 \%$ ), pericardial effusion (13 cases; $5.7 \%$ ), mitral regurgitation (24 cases; $4.8 \%$ ), tricuspid regurgitation (12 cases; $2.4 \%$ ), patent foramen ovale (11 cases; $2.2 \%$ ) and patent ductus arteriosus (6 cases; $1.2 \%$ ). In total, CAL, defined as ectasia or aneurysm, was observed in $40.9 \%$ of all cases undergoing echocardiographic examination. In certain patients, various cardiac abnormalities were identified simultaneously.

Therapies and prognosis. Treatment with intravenous immunoglobulin infusion (human freeze-dried low-pH intravenous gamma globulin; Biochemical Product Institute of Ministry of Public Health, Chengdu, China) was performed in 433 patients $(83.6 \%)$ within one month after KD onset. The regimens administered to patients included $400 \mathrm{mg} / \mathrm{kg} /$ day for 5 consecutive days in 6 cases $(1.4 \%), 1,000 \mathrm{mg} / \mathrm{kg}$ twice in 156 cases $(36.0 \%)$, $2,000 \mathrm{mg} / \mathrm{kg}$ once in 70 cases $(16.2 \%)$, and an irregular dose in 154 cases $(35.6 \%)$. Among these, the treatment regimen of $1,000 \mathrm{mg} / \mathrm{kg} /$ day twice was adopted more frequently. No adverse effects due to this treatment were identified in the 
current study. Besides intravenous immunoglobulin therapy, aspirin was administered orally in 455 cases, and steroids were administrated in 31 cases. In total, 310 patients were improved after treatment and were discharged from the hospital, whereas 14 patients were transferred to a higher level hospital and 194 patients were discharged without medical advice due to various limitations, such as financial difficulties and poorly conditioned local medical institutions.

Risk factors of CAL. Single-factor analysis of risk factors relevant to CAL incidence was conducted with a focus on patient age, gender, ethnic group, month of onset, duration of fever ( $\geq 5$ days), white blood cell count $\left(\geq 12 \times 10^{9} / 1\right)$, hemoglobin level $(<100 \mathrm{~g} / \mathrm{l})$, blood platelet count $\left(\geq 450 \times 10^{\%} / \mathrm{l}\right)$, erythrocyte sedimentation rate $(\geq 40 \mathrm{~mm} / \mathrm{h})$ and creatine kinase-MB level ( $\geq 25 \mathrm{U} / \mathrm{l}$ ). The results of the analysis indicated that ethnic group and month of onset were associated with the occurrence of CAL, as shown in Table I.

Furthermore, nonconditional multivariate logistic regression analysis was conducted for a single factor that was associated with CAL in KD. The Han Chinese ethnic group and July as the month of onset were selected for the multivariate logistic regression equation, and the corresponding P-values were found to be $<0.05$ (Table II). These results indicated that KD patients in the Han ethnic group were more likely to be complicated by CAL, whereas KD patients with onset occurring in July were less likely to be complicated by CAL. This may be due to climatic and environmental conditions, or living habits during different months, which may affect incidence; however, the possibility that this association was coincidental cannot be ruled out.

\section{Discussion}

KD was first recognized in 1967 (1); however, the etiology of the disease remains unclear. Various researchers have hypothesized that infectious agents may trigger the onset of $\operatorname{KD}(7,8)$, however, no certain conclusion can be reached. Therefore, a large-scale epidemiologic survey is required to identify clues regarding the etiology and pathogenesis of KD. Currently, KD has been reported in $>60$ countries and districts, including in Asia, Middle East, America, Africa and Europe (3). The incidence rate appears to be the highest in Japan, followed by East Asian countries and districts, such as China, Hong Kong, Taiwan, and Korea, with an increasing trend in numerous countries (3).

According to the results of the present 13-year survey conducted on KD cases between 2001 and 2013, the incidence rate was found to be increasing in Inner Mongolia during the study period, and the latest incidence rate was reported to be 7.7 per 100,000 children in 2013. The increasing trend has also previously been reported in other countries, districts and different regions of China (9-13). However, the awareness of this disease has increased among physicians during the time span, which may affect the accuracy of reported incidences of $\mathrm{KD}$. Therefore, continuous monitoring of KD is important to better understand the incidence trend of this disease.

Regarding the age distribution of patients at onset of KD, the present data demonstrated that the disease predominantly occurred in children under the age of 5 years, which accounted for $86.1 \%$ of the KD cases, and the peak age of KD onset was
$0-1$ years $(56.0 \%)$. These findings are in consistency with previous reports, while the male preponderance observed in the current study (male to female ratio, 1.64:1) was also comparable with previously studies from other regions of China (5,9-13).

As seasonal variation may be an important epidemiology characteristic of KD (3), the current study also focused on the seasonality of KD. According to the present results, the disease occurred all the year around, but more frequently in March, May to July, and November. The incidence appeared to peak in July, with the lowest point appearing in February. The seasonal distribution of KD occurrence in the present study is similar to that reported in a survey performed in Jilin province in China (5). However, great seasonal distribution discrepancies have been reported in studies involving different countries. For instance, in Japan, the number of patients was highest in January and lowest in October (14). In Korea, the majority of KD cases occurred in June, July and August, followed by December and January (15). In the United States, seasonal peaks in KD occurrence were observed in the winter and spring months (16), whereas in several European counties including England, Denmark and Ireland, there was a similar peak occurrence of KD in the winter months (17-19). Although seasonal distribution has been perceived as an important characteristic of descriptive epidemiology, the reasons for these discrepancies remain unclear. Different climatic and environmental conditions, ethnic group and living habits may possibly contribute to these discrepancies.

In consistency with previous studies (5,10-13), fever persisting for 5 days or longer was the most frequent clinical manifestation of KD, followed by changes in the lips and oral cavity, conjunctival congestion, polymorphous exanthema, changes in peripheral extremities and acute nonpurulent cervical lymphadenopathy. Notably, although perianal desquamation is not included in the diagnostic criteria of KD, it was observed in $22.8 \%$ of the cases included in the present study, and a relative higher incidence of perianal desquamation was also showed in several other studies $(5,11,20)$. As a systemic vasculitis, the present study also identified that KD affects multiple systems, including the respiratory and digestive system, and in certain cases, atypical clinical manifestations may lead to the misdiagnosis of KD (21); therefore, increased awareness of the spectrum of the clinical presentation of KD is essential for early diagnosis and treatment.

CAL is the most severe complication and a great health concern in KD patients. The incidence of CAL was reported to be $40.2 \%$ in the present survey during the acute stage, which was comparable with the rates reported by Baer et al (22) and Zhang et al (5). However, the rate reported in the present study was relatively high when compared with that of certain other reports $(9,11,13,16,23)$, which may be due to the delayed diagnosis and treatment as a result of the relatively lower economic level of the Inner Mongolia region. In addition, this inconsistency in CAL incidence may be due to differences in ethnicity and region, as well as discrepancy brought by multifarious diagnostic standards. Besides, regarding risk factors of CAL in patients with $\mathrm{KD}$, the present study indicated that patients in the Han ethnic group were more likely to be complicated by CAL, whereas KD patients whose month of onset was in July were less likely to be complicated by CAL; however, further 
studies are required to further investigate the underlying reasons for these observations.

It has been reported that intravenous immunoglobulin treatment is able to significantly relieve clinical symptoms and reduce the rate of CAL; therefore, it has become the main treatment for KD (24-27). In the current survey, $83.6 \%$ of the cases received treatment with intravenous immunoglobulin infusion within one month after KD onset. However, the optimal time window, dosage and the mechanism of the pharmacological effects of this treatment remain arguable $(27,28)$, and require further exploration.

There are certain limitations in the current study. Firstly, the retrospective nature of the study and the reliance on data from numerous different centers may affect the accuracy of the study. In addition, certain patients may not be included among the target hospitals for the survey due to misdiagnosis, economic limitations and lack of hospital space or of pediatric departments; the effects of these on the overall results are unlikely to be substantial.

In conclusion, the present study reported that the number of patients and incidence rate of KD in Inner Mongolia tend to increase year by year. The age distribution and clinical manifestations were similar to those reported in previous studies involving different region of China. Furthermore, the present study reported that the onset of KD was more frequent in spring and summer, and a higher incidence of CAL was reported in comparison with other studies. Ethnic group and month of onset were also found to be associated with the incidence of CAL in KD patients. Continues monitoring of KD and further studies focusing on identifying the etiology of KD are required to facilitate early diagnosis and more specific treatment of KD, therefore decreasing its morbidity and mortality rates.

\section{Acknowledgements}

The authors would like to thank all the hospitals and pediatricians participating in the present survey, and particularly thank the following hospitals: Maternal and Child Health Hospital of Inner Mongolia, Inner Mongolia Medical University Affiliated Hospital, Ordos Center Hospital, Chifeng City Hospital and Tongliao City Hospital.

\section{References}

1. Kawasaki T: Acute febrile mucocutaneous syndrome with lymphoid involvement with specific desquamation of the fingers and toes in children. Arerugi 16: 178-222, 1967 (In Japanese).

2. Ayusawa M, Sonobe T,Uemura S, Ogawa S, Nakamura Y, Kiyosawa $\mathrm{N}$ and Harada K; Kawasaki Disease Research Committee: Revision of diagnostic guidelines for Kawasaki disease (the 5th revised edition). Pediatr Int 47: 232-234, 2005.

3. Uehara R and Belay ED: Epidemiology of Kawasaki disease in Asia, Europe and the United States. J Epidemiol 22: 79-85, 2012.

4. Kuo HC, Yang KD, Chang WC, Ger LP and Hsieh KS: Kawasaki disease: An update on diagnosis and treatment. Pediatr Neonatol 53: 4-11, 2012

5. Zhang X, Zhang Z, Liu S and Sun J: Epidemiologic survey of Kawasaki disease in Jilin from 1999 through 2008. Pediatr Cardiol 33: 272-279, 2012.

6. Inner Mongolia Autonomous Region Bureau of Statistics. Inner Mongolia Statistical Yearbook 2001-2013. China Statistical Publishing House, Beijing, 2013.

7. Shibuya N, ShibuyaK, Kato Hand Yanagisawa M: Kawasakidisease before Kawasaki at Tokyo University Hospital. Pediatrics 110: e17, 2002 .
8. Nakamura Y, Yashiro M, Uehara R, Sadakane A, Chihara I, Aoyama Y, Kotani K and Yanagawa H: Epidemiologic features of Kawasaki disease in Japan: Results of the 2007-2008 nationwide survey. J Epidemiol 20: 302-307, 2010.

9. Du ZD, Zhao D, Du J, Zhang YL, Lin Y, Liu C and Zhang T; Beijing Kawasaki Research Group: Epidemiologic study on Kawasaki disease in Beijing from 2000 through 2004. Pediatr Infect Dis J 26: 449-451, 2007.

10. Huang WC, Huang LM, Chang IS, Chang LY, Chiang BL, Chen PJ, Wu MH, Lue HC and Lee CY: Epidemiologic features of Kawasaki disease in Taiwan, 2003-2006. Pediatrics 123: e401-e405, 2009.

11. Ma XJ, Yu CY, Huang M, Chen SB, Huang MR and Huang GY; Shanghai Kawasaki Research Group: Epidemiologic features of Kawasaki disease in Shanghai from 2003 through 2007. Chin Med J (Engl) 123: 2629-2634, 2010.

12. Li XH, Li XJ, Li H, Xu M and Zhou M: Epidemiological survey of Kawasaki disease in Sichuan province of China. J Trop Pediatr 54: 133-136, 2008.

13. Ng YM, Sung RY, So LY, Fong NC, Ho MH, Cheng YW, Lee SH, Mak WC, Wong DM, Yam MC, et al: Kawasaki disease in Hong Kong, 1994 to 2000. Hong Kong Med J 11: 331-335, 2005.

14. Nakamura Y, Yashiro M, Uehara R, Sadakane A, Tsuboi S, Aoyama Y, Kotani K, Tsogzolbaatar EO and Yanagawa H: Epidemiologic features of Kawasaki disease in Japan: Results of the 2009-2010 nationwide survey. J Epidemiol 22: 216-221, 2012.

15. Kim GB, Han JW, Park YW, Song MS, Hong YM, Cha SH, Kim DS and Park S: Epidemiologic features of Kawasaki disease in South Korea: Data from nationwide survey, 2009-2011. Pediatr Infect Dis J 33: 24-27, 2014.

16. Holman RC, Belay ED, Christensen KY, Folkema AM, Steiner CA and Schonberger LB: Hospitalizations for Kawasaki syndrome among children in the United States, 1997-2007. Pediatr Infect Dis J 29: 483-488, 2010.

17. Harnden A, Mayon-White R, Perera R, Yeates D, Goldacre M and Burgner D: Kawasaki disease in England: Ethnicity, deprivation and respiratory pathogens. Pediatr Infect Dis J 28: 21-24, 2009.

18. Fischer TK, Holman RC, Yorita KL, Belay ED, Melbye M and Koch A: Kawasaki syndrome in Denmark. Pediatr Infect Dis J 26: 411-415, 2007.

19. Lynch M, Holman RC, Mulligan A, Belay ED and Schonberger LB: Kawasaki syndrome hospitalizations in Ireland, 1996 through 2000. Pediatr Infect Dis J 22: 959-963, 2003.

20. Huang GY, Ma XJ, Huang M, Chen SB, Huang MR, Gui YH, Ning SB, Zhang TH, Du ZD, Yanagawa $\mathrm{H}$ and Kawasaki T: Epidemiologic pictures of Kawasaki disease in Shanghai from 1998 through 2002. J Epidemiol 16: 9-14, 2006.

21. Royle J, Burgner D and Curtis N: The diagnosis and management of Kawasaki disease. J Paediatr Child Health 41: 87-93, 2005.

22. Baer AZ, Rubin LG, Shapiro CA, Sood SK, Rajan S, Shapir Y, Romano A and Bierman FZ: Prevalence of coronary artery lesions on the initial echocardiogram in Kawasaki syndrome. Arch Pediatr Adolesc Med 160: 686-690, 2006.

23. Belay ED, Maddox RA, Holman RC, Curns AT, Ballah K and Schonberger LB: Kawasaki syndrome and risk factors for coronary artery abnormalities: United States, 1994-2003. Pediatr Infect Dis J 25: 245-249, 2006.

24. Newburger JW, Takahashi M, Beiser AS, Burns JC, Bastian J, Chung KJ, Colan SD, Duffy CE, Fulton DR and Glode MP: A single intravenous infusion of gamma globulin as compared with four infusions in the treatment of acute Kawasaki syndrome. N Engl J Med 324: 1633-1639, 1991.

25. Terai M and Shulman ST: Prevalence of coronary artery abnormalities in Kawasaki disease is highly dependent on gamma globulin dose but independent of salicylate dose. J Pediatr 131: 888-893, 1997.

26. Oates-Whitehead RM, Baumer JH, Haines L, Love S, Maconochie IK, Gupta A, Roman K, Dua JS and Flynn I: Intravenous immunoglobulin for the treatment of Kawasaki disease in children. Cochrane Database Syst Rev: CD004000, 2003.

27. Baba R, Shibata A and Tsurusawa M: Single high-dose intravenous immunoglobulin therapy for Kawasaki disease increases plasma viscosity. Circ J 69: 962-964, 2005.

28. Rigante D, Valentini P, Rizzo D, Leo A, De Rosa G, Onesimo R, De Nisco A, Angelone DF, Compagnone A and Delogu AB: Responsiveness to intravenous immunoglobulins and occurrence of coronary artery abnormalities in a single-center cohort of Italian patients with Kawasaki syndrome. Rheumatol Int 30: 841-846, 2010. 